\title{
Intracranial internal carotid artery calcification is not predictive of future cognitive decline
}

\author{
Farzaneh Rahmani ${ }^{1,2+}$, Marina Nguyen ${ }^{1,2+}{ }^{2}$, Charles D. Chen ${ }^{1,2}$, Nicole McKay ${ }^{1,2}$, Aylin Dincer ${ }^{1,2}$, \\ Nelly Joseph-Mathurin ${ }^{1,2}$, Gengsheng Chen ${ }^{1,2}$, Jingxia Liu ${ }^{3,4}$, Hilary L. P. Orlowski ${ }^{1}$, John C. Morris ${ }^{2,3}$ and \\ Tammie L. S. Benzinger ${ }^{1,2^{*}}$
}

\begin{abstract}
Background: Intracranial internal carotid artery (ICA) calcification is a common incidental finding in non-contrast head CT. We evaluated the predictive value of ICAC (ICAC) for future risk of cognitive decline and compared the results with conventional imaging biomarkers of dementia.
\end{abstract}

Methods: In a retrospective observational cohort, we included 230 participants with a PET-CT scan within 18 months of a baseline clinical assessment and longitudinal imaging assessments. Intracranial ICAC was quantified on baseline CT scans using the Agatson calcium score, and the association between baseline ICA calcium scores and the risk of conversion from a CDR of zero in baseline to a persistent CDR $>0$ at any follow-up visit, as well as longitudinal changes in cognitive scores, were evaluated through linear and mixed regression models. We also evaluated the association of conventional imaging biomarkers of dementia with longitudinal changes in cognitive scores and a potential indirect effect of ICAC on cognition through these biomarkers.

Results: Baseline ICA calcium score could not distinguish participants who converted to CDR $>0$. ICA calcium score was also unable to predict longitudinal changes in cognitive scores, imaging biomarkers of small vessel disease such as white matter hyperintensities (WMH) volume, or AD such as hippocampal volume, AD cortical signature thickness, and amyloid burden. Severity of intracranial ICAC increased with age and in men. Higher WMH volume and amyloid burden as well as lower hippocampal volume and AD cortical signature thickness at baseline predicted lower MiniMental State Exam scores at longitudinal follow-up. Baseline ICAC was indirectly associated with longitudinal cognitive decline, fully mediated through WMH volume.

Conclusions: In elderly and preclinical AD populations, atherosclerosis of large intracranial vessels as demonstrated through ICAC is not directly associated with a future risk of cognitive impairment, or progression of imaging biomarkers of $A D$ or small vessel disease.

Keywords: Internal carotid artery, Calcification, Clinical Dementia Rating, White matter hyperintensities, Mini-Mental State Exam, 11C-Pittsburgh compound B, PiB, Centiloid

*Correspondence: benzingert@wustl.edu

${ }^{\dagger}$ Farzaneh Rahmani and Marina Nguyen contributed as co-first authors.

${ }^{1}$ Mallinckrodt Institute of Radiology, Washington University School of Medicine, St. Louis, 510 South Kingshighway Boulevard, Campus Box 8131, St. Louis, MO 63110, USA

Full list of author information is available at the end of the article

\section{Introduction}

Mineralization of the intimal layer of the vessel wall is an integral part of the atherosclerotic process [1]. Calcification of the cervical internal carotid artery (ICA) is a original author(s) and the source, provide a link to the Creative Commons licence, and indicate if changes were made. The images or other third party material in this article are included in the article's Creative Commons licence, unless indicated otherwise in a credit line to the material. If material is not included in the article's Creative Commons licence and your intended use is not permitted by statutory regulation or exceeds the permitted use, you will need to obtain permission directly from the copyright holder. To view a copy of this licence, visit http://creativecommons.org/licenses/by/4.0/. The Creative Commons Public Domain Dedication waiver (http://creativeco mmons.org/publicdomain/zero/1.0/) applies to the data made available in this article, unless otherwise stated in a credit line to the data. 
well-studied example that is associated with the presence of cardiovascular risk factors and risk of stroke [2].

Intracranial ICAC (ICAC) is an expression of intracranial atherosclerosis and a common incidental finding on non-contrast computed tomography of the head (CT) [3]. Its prevalence ranges from 46 to $82 \%$ in the general adult population to almost $100 \%$ in individuals older than 90 years [4]. The most common sites of calcification are the cavernous carotid and the carotid siphon where the severity of calcification is associated with the presence of small vessel disease and white matter lesions [5-8], both of which have been shown to adversely affect cognition in older adults [9].

Studies have demonstrated an inverse relationship between the severity of intracranial ICAC and cognitive performance in terms of memory, executive function, global cognition, and processing speed in healthy adults $[10,11]$, as well as a relationship between extracranial ICAC and the risk of dementia [12]. Disrupted cerebral blood flow autoregulation, blood-brain barrier dysfunction, and increased amyloid deposition following increased ICA stiffness are among the suggested underlying mechanisms [13]. Little is known about the association of intracranial ICAC and Alzheimer disease (AD), in particular its association with imaging biomarkers of $\mathrm{AD}$, including $\beta$-amyloid deposition and cortical and hippocampal atrophy.

We conducted a retrospective cohort study on 230 participants to assess the relationship between intracranial ICAC and imaging biomarkers of $\mathrm{AD}$ and small vessel disease as well as cognitive outcomes. The primary objectives of the study were to investigate (1) whether there is a relationship between the presence and severity of baseline ICAC and risk of conversion from normal to impaired cognition and (2) whether ICAC can predict future cognitive outcomes through longitudinal analyses. As a secondary objective, we investigated (3) whether there is any association between baseline ICAC and longitudinal changes in imaging biomarkers of $\mathrm{AD}$ and small vessel disease and (4) whether there is any association between imaging biomarkers of $\mathrm{AD}$ and small vessel disease and longitudinal changes in cognitive scores. We used the latter results to further investigate any potential indirect effect of ICAC on cognition mediated through its effect on these imaging biomarkers.

\section{Methods}

\section{Participants}

Participants were selected from a cohort of individuals enrolled and recruited from February 2009 through March 2018 in the ongoing longitudinal studies of memory and aging at the Charles F. and Joanne Knight Alzheimer Disease Research Center (Knight ADRC) at the
Washington University School of Medicine in St. Louis. Inclusion criteria for this study were (1) having a PET-CT scan within 18 months of a clinical assessment, which was considered the baseline visit, and (2) having at least one follow-up clinical assessment. Among these, 52 participants were identified to convert from a Clinical Dementia Rating ${ }^{\mathrm{TM}}$ scale $\left(\mathrm{CDR}^{\mathrm{TM}}\right)$ [14] equal to 0 in their baseline visit to a CDR $>0$ in any of their follow-up assessments (case group or converters). A 2:1 age- and sex-matched control group was selected from among participants who had a $C D R=0$ at baseline and remained cognitively normal $(\mathrm{CDR}=0)$ in all follow-up assessments $(n=106)$. Finally, an impaired group was defined based on all participants who had a CDR $>0$ at their baseline visit $(n=$ 72 ). Figure 1 provides a summary of inclusion and exclusion criteria and how the final sample size was arrived at. To summarize, a total number of 230 participants (age range 52-90 years) were enrolled as part of the control ( $n$ $=106)$, converter $(n=52)$, and impaired $(n=72)$ groups. Exclusion criteria included (1) reverting back from CDR $>0$ to a $\mathrm{CDR}=0$ at any follow-up visit, (2) diagnosis of uncertain dementia, and (3) fluctuating between CDR $>0$ and $C D R=0$ at any time during follow-up visits. Figure 1 gives an overview on how participants in each group were selected and the number of participants excluded due to each reason.

Within the impaired group, 62 participants had a diagnosis of AD dementia defined based on a comprehensive clinical assessment including a detailed interview of a collateral source, a neurologic examination by a skilled physician, and the CDR score [15]. Also, 10 participants from the impaired group had AD dementia with co-morbid active major depressive disorder. None of the participants demonstrated atypical variants of AD including the logopenic (primary progressive aphasia) or posterior cortical atrophy variants. Among participants who converted, 37 participants had a diagnosis of AD dementia followed by 9 participants who scored 0.5 only in the memory domain of CDR, 3 participants with AD dementia and co-morbid active major depressive disorder, one participant with primary Parkinson disease dementia, one participant with frontotemporal dementia, and one participant with vascular dementia.

\section{Clinical and psychiatric assessments}

All participants underwent annual cognitive assessments which included a global CDR score evaluated by experienced clinicians utilizing a semi-structured participant interview and information from collateral sources. For longitudinal assessments of cognition, CDR Sum of Boxes score (CDR-SB), a summation of scores for each domain measured, was utilized [16]. Also included in the longitudinal cognitive assessment was the Mini-Mental 


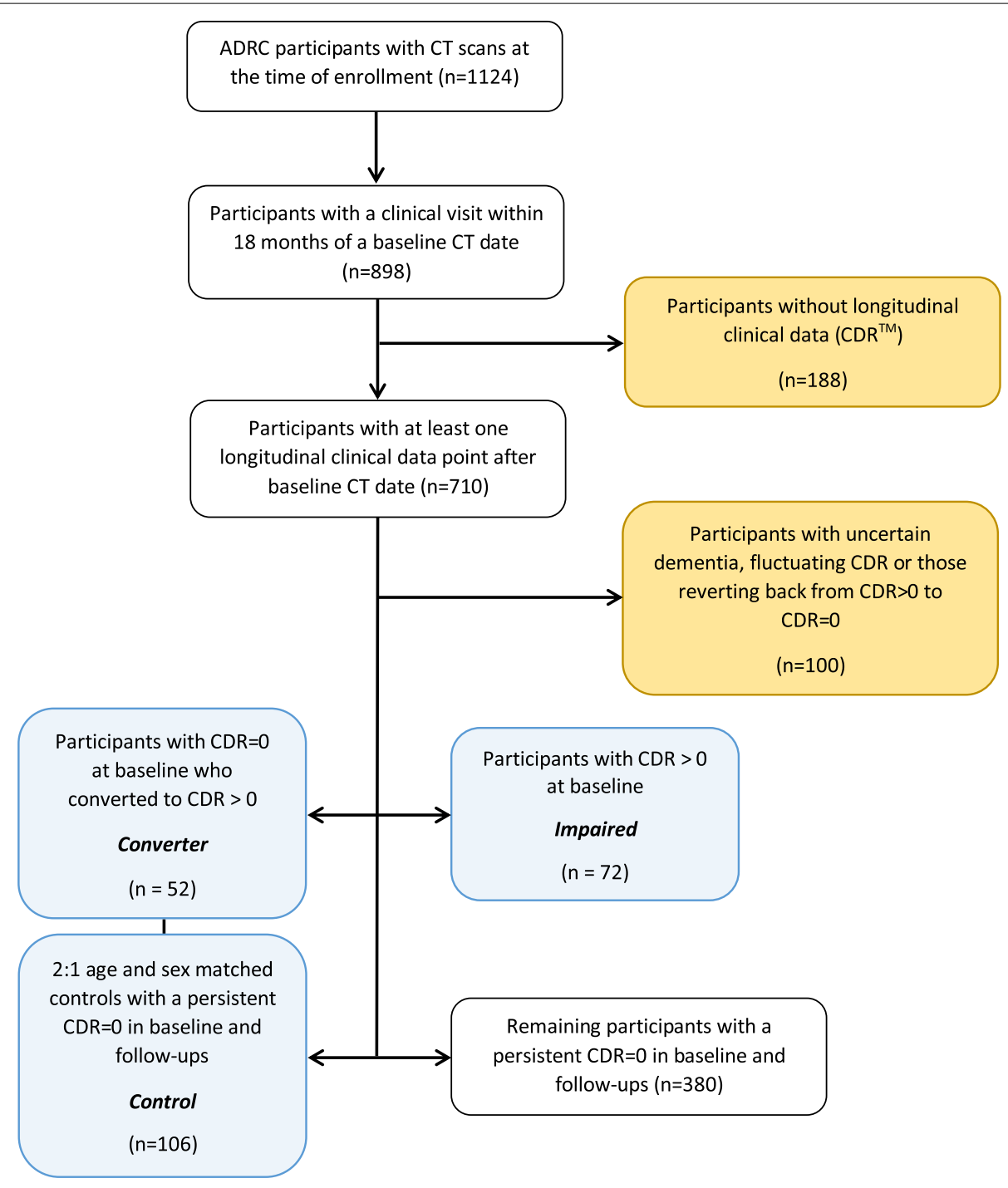

ADRC: Alzheimer disease research center; CDR: clinical dementia rating scale; CT: Computed Tomography

Fig. 1 Inclusion criteria for the study population and reasons for exclusion

State Examination (MMSE) score that was extracted from participants' annual clinical visits. Finally, we derived the Preclinical Alzheimer Cognitive Composite (PACC) score for each individual as a measure of overall cognitive performance from a subset of the Knight ADRC cognitive battery as described before [17].

\section{Imaging assessments}

\section{CT scan calcium scoring}

Non-contrast head CT scans were obtained using a Siemens Biograph 40 PET-CT scanner and transferred to a Vitrea 2 workstation (Vital Images Inc, Plymouth, MN). $\mathrm{CT}$ images were acquired using a tube voltage of $120 \mathrm{kV}$ and tube current-time of $22 \mathrm{mAs}$ in a $512 \times 512$ matrix. Scans all had 40 slices, included skull base to the vertex, had less than $0.6-\mathrm{mm}$ isotropic spatial resolution, and slice thickness of $3 \mathrm{~mm}$. Images were assessed for quality and absence of artifacts such as beam hardening or noise. Scans were reconstructed using the H19s algorithm and assessed using a bone window level $(\sim 1500$ Hounsfield Units). A semi-automated coronary calcium scoring software (CT VScore ${ }^{\mathrm{TM}}$, Cannon Medical Informatics) was used to calculate the Agatston calcium score and volume [18].

The training cohort was defined by randomly choosing $15 \%$ of the 230 participants. Once trained to the 
satisfaction of the board-certified neuroradiologist rater (H.O.), two raters (M.N. and F.R.) scored the entire set blindly and independently by drawing regions of interest (ROIs) around areas of ICAC starting from the distal petrous apex to the ICA terminus (cavernous, clinoid, ophthalmic, and communicating segments). Figure 2 demonstrates examples of ICA calcification in the cavernous and clinoid segments for two participants. For this purpose, Agatston calcium scores were divided into four categories based on the cut-off values used to classify coronary artery calcification in clinical practice [19]. According to this rating, a calcium score of 0 is considered as the absence of coronary calcification, while scores ranging from 1 to 100 are rated as discrete, 101 to 400 as moderate, and scores above 400 as accentuated. The raters achieved a two-way agreement intraclass correlation coefficient $(I C C)=0.845$ on raw calcium scores and an $I C C=0.889$ on calcium score categories. Categorization of ICA Agatston scores based on cut-off values established for coronary artery calcium scoring was only performed to calculate the ICC between raters. These categories were therefore not used in any of the statistical analyses that followed. $\mathrm{CT}$ scans that received scores that differed between the two raters to the extent of receiving scores that belonged to different categories were blindly scored a second time, and any remaining discordant scans received a final resolution score by the board-certified experienced rater (H.O.). As both ICA calcium score and volume had a non-normal distribution, the natural $\log (\mathrm{Ln})$-transformed values of both measures were used after adding one unit to the non-transformed values to deal with calcium scores of zero. Averaged scores between raters were used for analyses.

\section{Amyloid PET acquisition and processing}

Participants underwent amyloid PET imaging using either 11C-Pittsburgh compound B (PiB) or 18F-AV45

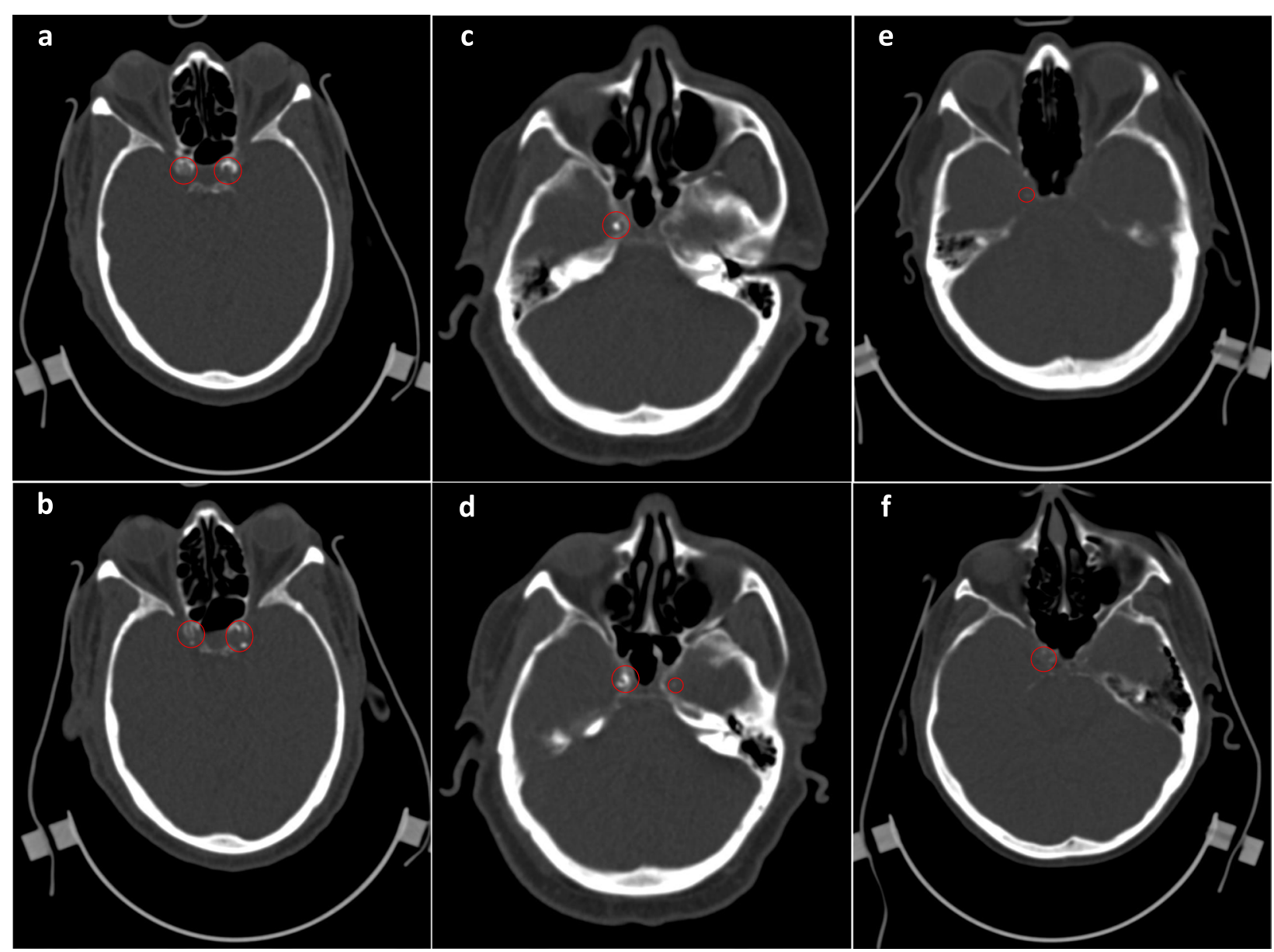

Fig. 2 Internal carotid artery (ICA) calcification in low resolution CT scans for two participants. a, b Participant A demonstrates calcifications in the carotid siphon (total Agatston score: 1256). In participant B (c, d), calcifications are demonstrated in the petrous (c) and cavernous (d) segments of the left ICA (total Agatston score: 763). Mild ICA calcification is demonstrated in e-f in participant C with a total Agatston score of 150 
(florbetapir) radioligands. Methods for $\mathrm{PiB}$ and AV45 PET acquisition have been described previously [20, 21]. All scans were obtained on a Siemens Biograph 40 PETCT or Biograph mMR and preprocessed using a unified longitudinal pipeline that outputs Centiloid values in order to standardize the PiB and AV45 tracers [22]. The amyloid PET data acquired on the day of the PET-CT scan was used to determine the amyloid status of all participants in their baseline. Amyloid positivity was defined as Centiloid values of $>16.4$ in all visits [22].

\section{MRI acquisition and processing}

White matter hyperintensities (WMH) volume, total hippocampal volume, and AD cortical signature thickness measurements were calculated for all baseline and longitudinal MRI assessments using the unified processing pipelines described below. T1- and T2-weighted images were acquired using a magnetization-prepared rapid gradient-echo sequence on the Siemens 3T TIM Trio or Biograph mMR scanners. T1-weighted images were acquired with a $1 \times 1 \times 1 \mathrm{~mm}$ resolution, $2400 \mathrm{~ms}$ repetition time, 3.16 echo time, $8^{\circ}$ flip angle, 176 frames, and a $256 \times$ 256 field of view in sagittal orientation, and T2-weighted images were acquired with a $1 \times 1 \times 1 \mathrm{~mm}$ resolution, $3200 \mathrm{~ms}$ repetition time, echo time of $455,120^{\circ}$ flip angle, and a $256 \times 256$ field of view.

White matter hyperintensity volume WMH volumes were calculated from a T2-weighted fluid-attenuated inversion recovery (FLAIR) and a $\mathrm{T} 1$ scan using the lesion segmentation toolbox (LST) implemented within SPM8 [23].

Total hippocampal volume and $A D$ cortical signature thickness Total hippocampal volumes were obtained on a T1-weighted image with the use of automated FreeSurfer 5.3. segmentation as previously described [24]. AD cortical signature thickness was also obtained from the T1-weighted image through an ROI cortical map representing the specific brain regions most susceptible to AD-related cortical atrophy as described previously [25].

\section{Statistical analysis}

Statistical analyses were performed using IBM SPSS Statistics version 27 and $\mathrm{R}$ version 4.1.1. Normality of distribution of all variables was tested through the Kolmogorov-Smirnov goodness-of-fit test. Baseline variables were compared between the three cognitive groups (control, converter, and impaired) using the ANOVA or Kruskal-Wallis tests depending on the presence or absence of a normal distribution for each variable, respectively. Similarly, comparisons between participants with and without ICAC were conducted using the independent $t$-test or Mann-Whitney $U$ test for variables with and without a normal distribution respectively. Correction for multiple comparisons using the false discovery rate (FDR) through the Benjamini-Hochberg method was performed when appropriate [26].

The binary logistic regression model was used to evaluate the association of baseline variables with the odds of conversion to CDR above 0 (odds of conversion $\sim \mathrm{Ln}$ transformed ICA calcium score/volume, age, cognitive scores in baseline, biomarkers of $\mathrm{AD}$, and small vessel disease in baseline) as well as the odds of ICAC (odds of the presence of ICAC biomarkers of AD and small vessel disease in baseline, age). Univariable analyses were conducted first to identify variables with significant association with the outcome that were then entered into a multivariable analysis through stepwise selection [27]. A significance level of 0.3 was required to allow a predictor into the multivariable model and a significance level of 0.1 was required for a predictor to stay in the model where variables were selected through a backward likelihood ratio (LR) method in the multivariable model. Type I error probability of 0.05 was used to identify significant variables in all regression models. Survival analyses using the Cox proportional hazards $(\mathrm{P}-\mathrm{H})$ model were also used to investigate the effect of ICAC in different levels of baseline amyloid status with conversion to CDR $>0$ as outcome (odds of conversion ICA calcification*amyloid status). The $\mathrm{P}-\mathrm{H}$ assumption was tested through adding a time-dependent variable to the model.

For longitudinal analyses, the $\mathrm{R}$ packages "lmerTest" and "lme4" were used to run a linear mixed model to extract the estimated annual rate of change in cognitive scores, WMH volume, and AD imaging biomarkers in the whole cohort including the control, converter, and impaired groups. Longitudinal measures of the variables of interest were considered dependent variables while time from baseline visit (time) and observations overtime for each subject (time | subject) were considered as fixed factors. Next, a simple linear model was devised to investigate the association between baseline Ln-transformed ICA calcium score and volume as an independent variable and estimated annual rates of change in cognitive scores and biomarkers of $\mathrm{AD}$ and small vessel disease (estimated annual rate of change in MMSE, PACC, CDR$\mathrm{SB}, \mathrm{AD}$, and small vessel disease biomarkers $\sim$ baseline Ln ICA calcium score/volume). A similar model was built to investigate the association between baseline biomarkers of AD and small vessel disease and estimated annual rate of change in MMSE and PACC score as dependent variables (estimated annual rate of change in MMSE and PACC score biomarkers of AD and small vessel disease 
in baseline). Finally, the estimated marginal means of the dependent variables were plotted against time across different levels of the fixed effect of interest. Type I error probability of 0.05 was used to identify significant variables in all regression models.

Regression models described above allowed us to investigate the potential association of ICA calcium score/volume and imaging biomarkers of dementia and small vessel disease with longitudinal changes in cognitive scores. As a final step, we investigated any potential indirect effect of ICAC on longitudinal changes in cognition that was mediated through its effect on other relevant biomarkers. We used the R package "mediation" and implemented a design-based approach considering ICA calcium score or volume as exposure and longitudinal cognitive scores as outcome [28]. The annual rate of change in MMSE score was estimated for each participant using the method described for the longitudinal analyses and was considered as a dependent variable. The Ln-transformed ICA calcium score was considered an independent variable and WMH volume, hippocampal volume, AD cortical signature thickness, and Centiloid values were adopted as mediators in the model one at a time. The significance of each mediation model was tested using 1000 bootstrapped samples and considering a type I error probability of 0.05 as a significance level.

\section{Results}

No difference in ICA calcification, WMH volume, or AD imaging biomarkers between converters and controls

Figure 1 demonstrates a flow diagram showing the initial number of potentially eligible participants; selection criteria for the control, converter, and impaired groups; and those excluded for different reasons. A description of demographic, clinical, and cognitive risk factors of different groups in the baseline is presented in Table 1. There was no statistically significant difference between the control and converter groups in baseline calcium scores or volumes, WMH volume, or AD imaging biomarkers. The impaired group however had a higher WMH volume

Table 1 Demographics, cognitive status, vascular risk factors, and AD imaging biomarkers of participant groups in the baseline

\begin{tabular}{|c|c|c|c|c|c|}
\hline & Total $(n=230)$ & Control $(n=106)$ & Converter $(n=52)$ & Impaired $(n=72)$ & $p$-value $e^{\S \S}$ \\
\hline Age, years (mean $\pm S D^{\S}$ ) & $73.7 \pm 6.7$ & $73.2 \pm 6.6$ & $73.1 \pm 6.7$ & $74.8 \pm 6.9$ & 0.418 \\
\hline \multicolumn{6}{|l|}{ Sex } \\
\hline Men (n (\%)) & $121(52.6)$ & $54(50.9)$ & $26(50)$ & $41(56.9)$ & \multirow[t]{2}{*}{0.669} \\
\hline Women (n (\%)) & $109(47.7)$ & $52(49.1)$ & $26(50)$ & $31(43.1)$ & \\
\hline \multicolumn{6}{|l|}{ Race } \\
\hline Caucasian (n (\%)) & $197(85.6)$ & $93(87.8)$ & $43(82.7)$ & $61(84.7)$ & \multirow[t]{4}{*}{0.466} \\
\hline African-American (n (\%)) & $25(10.9)$ & $11(10.4)$ & $6(11.5)$ & $8(11.2)$ & \\
\hline Native American (n (\%)) & $1(<1)$ & $0(0)$ & $1(1.9)$ & $0(0)$ & \\
\hline Unknown (n (\%)) & $7(3)$ & $2(1.8)$ & $2(3.9)$ & $3(4.1)$ & \\
\hline MMSE score $(\text { median }(\mathrm{Q} 1-\mathrm{Q} 3))^{\S}$ & $29(28-30)$ & $29(29-30)$ & $29(28-30)^{\# \# \#}$ & $28(26-29)^{* * *}$ & $<0.001$ \\
\hline CDR-SB (median (min-max)) & $0(1)$ & $0(0-0.5)$ & $0(0-0.5)^{\# \# \#}$ & $1.5(0.5-6)^{* * *}$ & $<0.001$ \\
\hline PACC score (median (Q1-Q3)) & $0.04(-0.21-0.25)$ & $0.13(-0.03-0.41)$ & $0.084(-0.15-0.23)^{\#}$ & $-0.23(-0.41-0.04)^{* * *}$ & $<0.001$ \\
\hline WMH volume, $\mathrm{mm}^{3}$ (mean \pm SD) & $16,072 \pm 24,128$ & $18,024 \pm 19,131$ & $21,819 \pm 18,562$ & $29,603 \pm 26,163^{* *}$ & 0.005 \\
\hline Ln ICA Ca score (median (Q1-Q3)) & $3.2(0-4.8)$ & $3.4(0.69-4.8)$ & $2.9(0-4.05)$ & $3.2(0-5.2)$ & 0.349 \\
\hline Ln ICA Ca volume (median (Q1-Q3)) & $3.7(0-4.9)$ & $3.94(1.55-4.96)$ & $3.6(0-4.4)$ & $3.5(0-5.2)$ & 0.520 \\
\hline Total hippocampal volume, $\mathrm{mm}^{3}$ (mean \pm SD) & $7004 \pm 967.6$ & $7397 \pm 793$ & $6991 \pm 844^{\#}$ & $6397 \pm 1008^{* * *}$ & $<0.001$ \\
\hline AD cortical signature thickness, mm (mean $\pm S D$ ) & $2.5 \pm 0.15$ & $2.5 \pm 0.12$ & $2.5 \pm 0.13^{\#}$ & $2.4 \pm 0.17^{* * *}$ & $<0.001$ \\
\hline Centiloid (mean \pm SD) & $6.9(69.9)$ & $3.4 \pm 35.3$ & $66.5 \pm 83.2$ & $15.3 \pm 75.1^{* * *}$ & $<0.001$ \\
\hline Amyloid positivity (negative/positive) & $114 / 116$ & $70 / 36$ & $23 / 29$ & $21 / 51$ & $<0.001$ \\
\hline
\end{tabular}

Asterisks represent significant post hoc tests following the Kruskal-Wallis or ANOVA models: ${ }^{*}<0.05,{ }^{* *}<0.005$, and ${ }^{* * *}<0.0001$ represent post hoc models showing the significant difference from the control group while ${ }^{\#}<0.05,{ }^{\# \#}<0.005$, and ${ }^{\# \# \#}<0.0001$ show significant difference from the impaired group

Abbreviations: Control, participants with CDR $=0$ throughout all visits, Converter: participants converting from $C D R=0$ to $C D R>0$ in any of the follow-up visits, Impaired, participants with CDR > 0 in the baseline visit, MMSE, Mini-Mental State Examination, CDR, Clinical Dementia Rating Scale, CDR-SB, Clinical Dementia Rating Scale Sum of Boxes, PACC, Preclinical Alzheimer Cognitive Composite, WMH volume, white matter hyperintensities volume, AA, African-American, C, Caucasian, Nat, Native American, U, unknown race, Ln ICA Ca score/volume, natural log-transformed internal carotid artery Agatston calcium score/volume, $A D$ cortical signature thickness, cortical thickness in signature regions affected in Alzheimer disease (see Dincer et al. 2020), Centiloid, measure of global amyloid disposition based on the conversion of PIB or AV45 PET SUVRs to a standardized scale. Amyloid positivity was defined based on Centiloid values > 16.4

${ }^{\S}$ Data is reported in form of mean \pm standard deviation (SD) for normally distributed variables and as median plus the first and third quartiles (Q1-Q3) for variables without normal distribution. Normality was determined using the Kolmogorov-Smirnov goodness-of-fit test

${ }^{\$ \varsigma}$ Kruskal-Wallis test was used to compare ICA Ca score and volume, MMSE, and PACC scores between the three baseline groups. Comparison for all other variables was conducted using an ANOVA model. Bold values indicate the test with statistical significance considering a threshold of 0.05 
and Centiloid values as well as lower AD cortical signature thickness and total hippocampal volume compared to the control and converter groups. The presence of ICA was not associated with any significant difference in cognitive scores or AD imaging biomarkers. Men had more severe ICAC compared to women (Table 2).

\section{Amyloid burden, but not ICA calcification, is associated with future risk of cognitive decline}

According to existing literature, we investigated the association of hippocampal volume [29], cortical thickness [30], and amyloid burden [31] with the risk of conversion to $\mathrm{CDR}>0$. Using univariable logistic regression models, lower hippocampal volume, and higher Centiloid values, but neither Ln-transformed ICA calcium score nor volume was found to be associated with higher odds of conversion to CDR above zero (Table 3). In the multivariable regression model, lower total hippocampal volume and higher Centiloid values were independently associated with higher odds of conversion to CDR above zero (Table 3) (overall model $R$-square: $0.245, p$-value $<0.001$ ).

\section{Severity of white matter disease and increased age predict ICAC at baseline}

As neither Ln ICA calcium score nor Ln ICA calcium volume had a normal distribution, we investigated the odd of the presence of ICAC-using a cut-off of 0 or odds of high versus low ICA calcium score-using the median value for Ln ICA calcium score/volume as cut-off-as dependent variables in our regression model. Predictors were selected according to literature and included age and sex [32], AD imaging biomarkers [33], and imaging biomarkers of small vessel disease [6]. Increased age, male sex, and decreased AD cortical signature thickness were associated with the presence and severity of ICAC, and lower AD cortical thickness, and higher WMH volume were associated with the ICAC severity (Table 4). In the multivariable

Table 2 Demographics, cognitive status, and AD imaging biomarkers of participants with and without internal carotid artery calcification in the baseline

\begin{tabular}{|c|c|c|c|}
\hline & Calcification absent $(n=64)$ & Calcification present $(n=166)$ & $p$-value ${ }^{\S \S}$ \\
\hline Control/converter/impaired ( $n$ ) & $25 / 15 / 24$ & $81 / 37 / 48$ & 0.356 \\
\hline Age, years (mean $\pm S D)$ & $74.4 \pm 6.8$ & $74.9 \pm 4.2$ & 0.862 \\
\hline \multicolumn{4}{|l|}{ Sex } \\
\hline Men (n (\%)) & $22(34.3)$ & $99(59.6)$ & \multirow[t]{2}{*}{0.001} \\
\hline Women $(n(\%))$ & $42(65.7)$ & $67(40.4)$ & \\
\hline \multicolumn{4}{|l|}{ Race } \\
\hline Caucasian (n (\%)) & $50(78.1)$ & $147(88.6)$ & \multirow[t]{4}{*}{0.110} \\
\hline African-American ( $(\%))$ & $12(18.7)$ & 13(7.8) & \\
\hline Native American (n (\%)) & $0(0)$ & $1(0.6)$ & \\
\hline Unknown (n (\%)) & $2(3.1)$ & $5(3)$ & \\
\hline MMSE (median (Q1-Q3)) $)^{\S}$ & $29(27-30)$ & $29(28-30)$ & 0.061 \\
\hline CDR-SB (median (IQR)) & $0(0.5)$ & $0(1)$ & 0.38 \\
\hline PACC score (median (Q1-Q3)) $)^{\S}$ & $0.039(-0.23-0.23)$ & $0.041(-0.16-0.27)$ & 0.334 \\
\hline WMH volume, $\mathrm{mm}^{3}($ mean $\pm \mathrm{SD})$ & $15841 \pm 14220)$ & $24424 \pm 23447$ & 0.173 \\
\hline Total hippocampal volume, $\mathrm{mm}^{3}$ (mean \pm SD) & $7052 \pm 878$ & $7203 \pm 890$ & 0.727 \\
\hline AD cortical signature thickness, mm (mean \pm SD) & $2.5 \pm 0.17$ & $2.5 \pm 0.11$ & 0.771 \\
\hline Centiloid (mean \pm SD) & $15.3 \pm 73.4$ & $5.3 \pm 75.9$ & 0.502 \\
\hline Amyloid positivity (negative/positive) & $30 / 34$ & $84 / 82$ & 0.660 \\
\hline
\end{tabular}

Asterisks represent significant post hoc tests following the Kruskal-Wallis or ANOVA models: ${ }^{*}<0.05,{ }^{* *}<0.005$, and ${ }^{* *}<0.0001$ represent post hoc models showing a significant difference from the control group while ${ }^{\#}<0.05,{ }^{\#}<0.005$, and ${ }^{\# \# \#}<0.0001$ show significant difference from the impaired group

Abbreviations: Control, participants with CDR $=0$ throughout all visits, Converter, participants converting from $C D R=0$ to $C D R>0$ in any of the follow-up visits, Impaired, participants with CDR $>0$ in the baseline visit, MMSE, Mini-Mental State Examination, CDR, Clinical Dementia Rating Scale, CDR-SB, Clinical Dementia Rating Scale Sum of Boxes, PACC, Preclinical Alzheimer Cognitive Composite, WMH volume, white matter hyperintensities volume, $A A$, African-American, $C$, Caucasian, Nat, Native American, U, unknown race, Ln ICA Ca score/volume, natural log-transformed internal carotid artery Agatston calcium score/volume, AD cortical signature thickness, cortical thickness in signature regions affected in Alzheimer disease (see Dincer et al. 2020), Centiloid, measure of global amyloid disposition based on conversion of PIB or AV45 PET SUVRs to a standardized scale. Amyloid positivity was defined based on Centiloid values $>16.4$

${ }^{\S}$ Data is reported in form of mean \pm standard deviation (SD) for normally distributed variables and as median plus the first and third quartiles (Q1-Q3) for variables without normal distribution. Normality was determined using the Kolmogorov-Smirnov goodness-of-fit test

$\S \S$ Mann-Whitney $U$ test was used to compare MMSE and PACC scores between the two groups. Comparison for all other variables was conducted using an independent samples $t$-test. Bold values indicate test with statistical significance considering a threshold of 0.05 
Table 3 Binary logistic regression model to predict the odds of conversion to CDR above zero based on baseline biomarkers

\begin{tabular}{|c|c|c|c|c|}
\hline & \multicolumn{4}{|c|}{ Odds of conversion from $C D R=0$ to $C D R>0$} \\
\hline & \multicolumn{2}{|c|}{ Univariable } & \multicolumn{2}{|c|}{ Multivariable } \\
\hline & p-value & OR $(95 \% \mathrm{Cl})$ & $p$-value & $O R^{\mathrm{b}}(95 \% C l)$ \\
\hline Age (years) & 0.996 & $1(0.951-1.051)$ & - & - \\
\hline Sex (male versus female) & 0.911 & $0.963(0.496-1.87)$ & - & - \\
\hline Ln ICA calcium score & 0.210 & $0.891(0.743-1.068)$ & - & - \\
\hline Ln ICA calcium volume & 0.265 & $0.899(0.845-1.084)$ & - & - \\
\hline WMH volum ${ }^{\mathrm{a}}\left(1000 \mathrm{~mm}^{3}\right)$ & 0.340 & $1.01(0.989-1.032)$ & - & - \\
\hline Total hippocampal volume $\left(100 \mathrm{~mm}^{3}\right)$ & 0.006 & $0.940(0.9-0.982)$ & 0.071 & $0.958(0.915-1.004)$ \\
\hline AD cortical signature thickness (mm) & 0.125 & $0.110(0.007-1.837)$ & - & - \\
\hline Centiloid $^{\mathrm{a}}$ (5 unit) & 0.003 & $1.08(1.027-1.136)$ & 0.012 & $1.070(1.015-1.127)$ \\
\hline
\end{tabular}

Abbreviations: ICA, internal carotid artery, WMH volume, white matter hyperintensities volume, Centiloid, measure of global amyloid disposition based on conversion of PIB or AV45 PET SUVRs to a standardized scale, AD cortical signature thickness, cortical thickness in signature regions affected in Alzheimer disease (see Dincer et al. 2020), Ln ICA calcium score/volume, natural log transformation of internal carotid artery Agatston calcium score/volume, OR (95\% CI), odds ratio and $95 \%$ confidence interval

${ }^{a}$ Odds ratios are demonstrated for $1000-\mathrm{mm}^{3}$ increments in WMH volume, $100-\mathrm{mm}^{3}$ increments in total hippocampal volume, and 5-unit increments in Centiloid scale

${ }^{b}$ Significant variables from the univariable model were entered into a stepwise multivariable model where a probability of 0.3 was set to enter the variables into the model and a probability of 0.1 to remove variables from the model

${ }^{\S} p$-values were corrected for multiple comparisons through estimation of the false discovery rate via the Benjamini and Hochberg method. Variables with a $p$-value of 0.05 were considered statistically significant in both regression models (denoted in bold)

regression model, age was the only independent predictor of the presence of ICAC (Table 4).

Similar to the results from the binary logistic regression analyses described in the previous section, survival analyses using the $\mathrm{P}-\mathrm{H}$ cox model revealed the absence of any association between ICAC in the baseline and risk of conversion (hazard ratio $(H R)(95 \%$ $C I): 0.866(0.474-1.579), p$-value $=0.638)$, while amyloid positivity (using a 16.4 cut-off for Centiloid values) was associated with a risk of conversion to CDR $>0(H R(95 \% C I): 2.3(1.3-4.1), p$-value $=0.04)$. There was no interaction between amyloid status and ICAC in predicting the conversion risk (HR $(95 \% \mathrm{CI}): 0.826$ $(0.242-2.817), p$-value $=0.760)$.

ICAC is not predictive of longitudinal changes in cognition, WMH volume, or AD imaging biomarkers

Table 5 summarizes the number of patients and sessions used in each set of longitudinal biomarker analyses. Longitudinal analyses were all performed using the full cohort including the control, converter, and impaired groups. Univariable regression models were applied to evaluate the association of baseline variables with annual rates of change in cognitive scores and imaging biomarkers, estimated through a linear mixed model. Ln-transformed ICA calcium score or volume was not associated with any difference in the annual rate of change of MMSE, CDR-SB, or PACC score; WMH volume; total hippocampal volume; AD cortical signature thickness; or Centiloid values (Fig. 3 and Table 6). On the other hand, higher WMH volume and amyloid burden in the baseline as well as lower baseline hippocampal volume and AD cortical signature thickness were associated with a steeper rate of annual decline in MMSE and PACC scores (Table 7).

\section{Effect of baseline ICAC on cognitive decline is fully mediated by white matter disease}

Mediation analyses were used to investigate the presence of any potential indirect effect of ICAC on longitudinal cognition. Consistent with analyses described above, Ln-transformed ICA calcium score or volume was not significantly associated with longitudinal MMSE scores. However, mediation analyses revealed an indirect effect of baseline Ln-transformed ICA calcium score or volume on longitudinal MMSE, mediated solely by baseline WMH volume. As Fig. 4 illustrates, the regression model between baseline Ln-transformed ICA calcium score and WMH volume as well as the regression model between baseline WMH volume and estimated annual rate of change in MMSE was significant. The indirect effect of one unit increment in Ln-transformed ICA calcium score on the estimated annual rate of change in MMSE was therefore estimated to be -0.035 ( $95 \% C I$ : $[-0.06 ;-0.02]$, $p$-value $<0.001)$. Similarly, the indirect effect of one unit increase in Ln-transformed ICA calcium volume on the estimated annual rate of change in MMSE was estimated 
Table 4 Linear regression model to explore baseline variables with statistically significant associations with ICA Calcification in baseline

\begin{tabular}{|c|c|c|c|c|}
\hline & \multicolumn{4}{|c|}{ Presence vs. absence of ICA calcification ${ }^{\S \S}$} \\
\hline & \multicolumn{2}{|c|}{ Univariable } & \multicolumn{2}{|c|}{ Multivariable } \\
\hline & $p$-value & OR $(95 \% \mathrm{Cl})$ & $p$-value & $O R(95 \% C l)^{b}$ \\
\hline Age (years) & $<0.001$ & $1.136(1.08-1.195)$ & $<0.001$ & $1.11(1.05-1.175)$ \\
\hline Sex (male versus female) & $<0.001$ & $2.82(1.54-5.14)$ & 0.078 & $1.9(1.03-2.8)$ \\
\hline WMH volume $\left(1000 \mathrm{~mm}^{3}\right)^{\mathrm{a}}$ & 0.058 & $1.025(1.002-1.049)$ & - & - \\
\hline Total hippocampal volume $\left(100 \mathrm{~mm}^{3}\right)^{\mathrm{a}}$ & 0.489 & $0.989(0.958-1.02)$ & - & - \\
\hline AD cortical signature thickness (mm) & 0.022 & $0.053(0.006-0.480)$ & 0.582 & $0.499(0.042-5.92)$ \\
\hline \multirow[t]{4}{*}{ Centiloid (5 units) ${ }^{\mathrm{a}}$} & 0.49 & $0.985(0.952-1.02)$ & - & - \\
\hline & \multicolumn{4}{|c|}{ High vs. low ICA calcification ${ }^{\S \S}$} \\
\hline & \multicolumn{2}{|c|}{ Univariable } & \multicolumn{2}{|c|}{ Multivariable } \\
\hline & $p$-value & $O R(95 \% C l)$ & $p$-value & $O R(95 \% C l)^{b}$ \\
\hline Age (years) & $<0.001$ & $1.11(1.06-1.164)$ & 0.048 & $1.085(1.015-1.159)$ \\
\hline Sex (male versus female) & 0.005 & $2.4(1.40-4.16)$ & 0.168 & $1.6(0.807-3.42)$ \\
\hline WMH volume $\left(1000 \mathrm{~mm}^{3}\right)^{\mathrm{a}}$ & 0.005 & $1.030(1.011-1.048)$ & 0.163 & $1.012(0.991-1.033)$ \\
\hline Total hippocampal volume $\left(100 \mathrm{~mm}^{3}\right)^{a}$ & 0.277 & $0.984(0.956-1.012)$ & - & - \\
\hline AD cortical signature thickness (mm) & 0.006 & $0.056(0.008-0.397)$ & 0.262 & $0.964(0.921-1.0080)$ \\
\hline Centiloid (5 units) ${ }^{\mathrm{a}}$ & 0.277 & $0.982(0.950-1.015)$ & - & - \\
\hline
\end{tabular}

Abbreviations: ICA, internal carotid artery, WMH volume, white matter hyperintensities volume, Centiloid, measure of global amyloid disposition based on conversion of PIB or AV45 PET SUVRs to a standardized scale, AD cortical signature thickness, cortical thickness in signature regions affected in Alzheimer disease (see Dincer et al. 2020), OR (95\% CI), odds ratio and $95 \%$ confidence interval; the presence or absence of ICA calcification was determined based on natural log transformation of ICA calcium score/volume

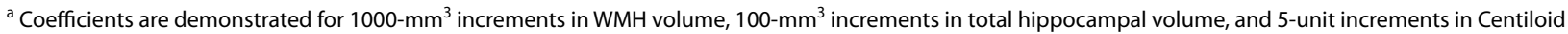
scale

${ }^{b}$ Significant variables from the univariable model were entered into a stepwise multivariable model where a probability of 0.3 was set to enter the variables into the model and a probability of 0.1 to remove variables from the model

$\S p$-values were corrected for multiple comparisons through estimation of the false discovery rate via the Benjamini and Hochberg method. Tests with a $p$-value of 0.05 were considered statistically significant in both regression models (denoted in bold).

$\S \S$ Presence of ICA calcification was defined based on Agatston ICA calcium score/volume using a cut-off of 0 . High versus low ICA calcification was determined using the median value for natural log transformation of ICA calcium score/volume as cut-off

as $-0.034(95 \% C I$ : $[-0.05 ;-0.01], p$-value $<0.001)$. In a similar set of analyses, total hippocampal volume, AD cortical signature thickness, and Centiloid values were unable to mediate any indirect effect between ICA calcium score or volume and longitudinal MMSE scores.

\section{Discussion}

We conducted a retrospective cohort study on 230 adults from the Knight ADRC cohort to investigate the relationship between intracranial ICAC and cognitive outcomes, as well as imaging biomarkers of $\mathrm{AD}$ and small vessel

Table 5 Participants and session characteristics contributing to each set of longitudinal visits and variables

\begin{tabular}{|c|c|c|c|c|c|c|}
\hline Visit type & Variables involved & $\begin{array}{l}\text { Total } \\
\text { included } \\
\text { visits }(n)\end{array}$ & $\begin{array}{l}\text { Number of } \\
\text { participants } \\
(n)\end{array}$ & $\begin{array}{l}\text { Between visit gap, } \\
\text { month (mean } \pm S D \text { ) }\end{array}$ & $\begin{array}{l}\text { Number of visits } \\
\text { (median (lower-upper } \\
\text { quartile)) }\end{array}$ & $\begin{array}{l}\text { Total } \\
\text { person-time } \\
\text { (months) }\end{array}$ \\
\hline Clinical & CDR-SB/MMSE & $1316 / 1257$ & 230 & $14.5 \pm 6.7$ & $3(2-5)$ & 9902 \\
\hline Psychometric & PACC score & 1008 & 229 & $14.4 \pm 6.6$ & $3(2-5)$ & 25018 \\
\hline MR session & $\begin{array}{l}\text { AD cortical signature thick- } \\
\text { ness/Total Hippocampal } \\
\text { volume }\end{array}$ & $341 / 341$ & 118 & $25.5 \pm 18.3$ & $2(1-3)$ & 14509 \\
\hline WMH session & WMH volume & 214 & 83 & $30.6 \pm 16.8$ & $2(1-2)$ & 5210 \\
\hline PIB/AV45 PET session & Centiloid & 102 & 46 & $40.8 \pm 21.3$ & $2(1-2)$ & 10699 \\
\hline
\end{tabular}

Abbreviations: MMSE, Mini-Mental State Examination, CDR, Clinical Dementia Rating Scale, CDR-SB, Clinical Dementia Rating Scale Sum of Boxes, PACC, Preclinical Alzheimer Cognitive Composite, WMH, white matter hyperintensities, PIB, Pittsburgh compound B, AV45, $18 \mathrm{~F}-\mathrm{AV} 45$ (florbetapir), Centiloid, measure of global amyloid disposition based on the conversion of PIB or AV45 PET total cortical standardized uptake ratios to a standardized scale with arbitrary units ranging from 0 to 100 , Total Hipp, total hippocampal volume, AD cortical signature thickness, cortical thickness in signature regions affected in Alzheimer disease 


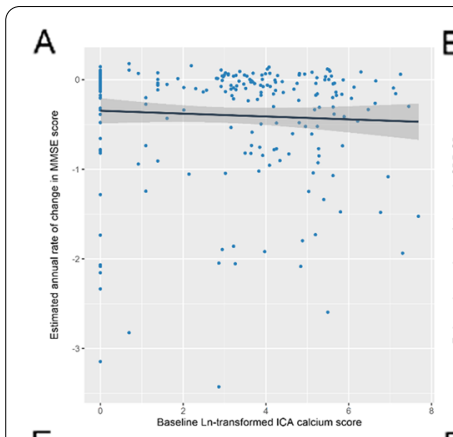

$E$
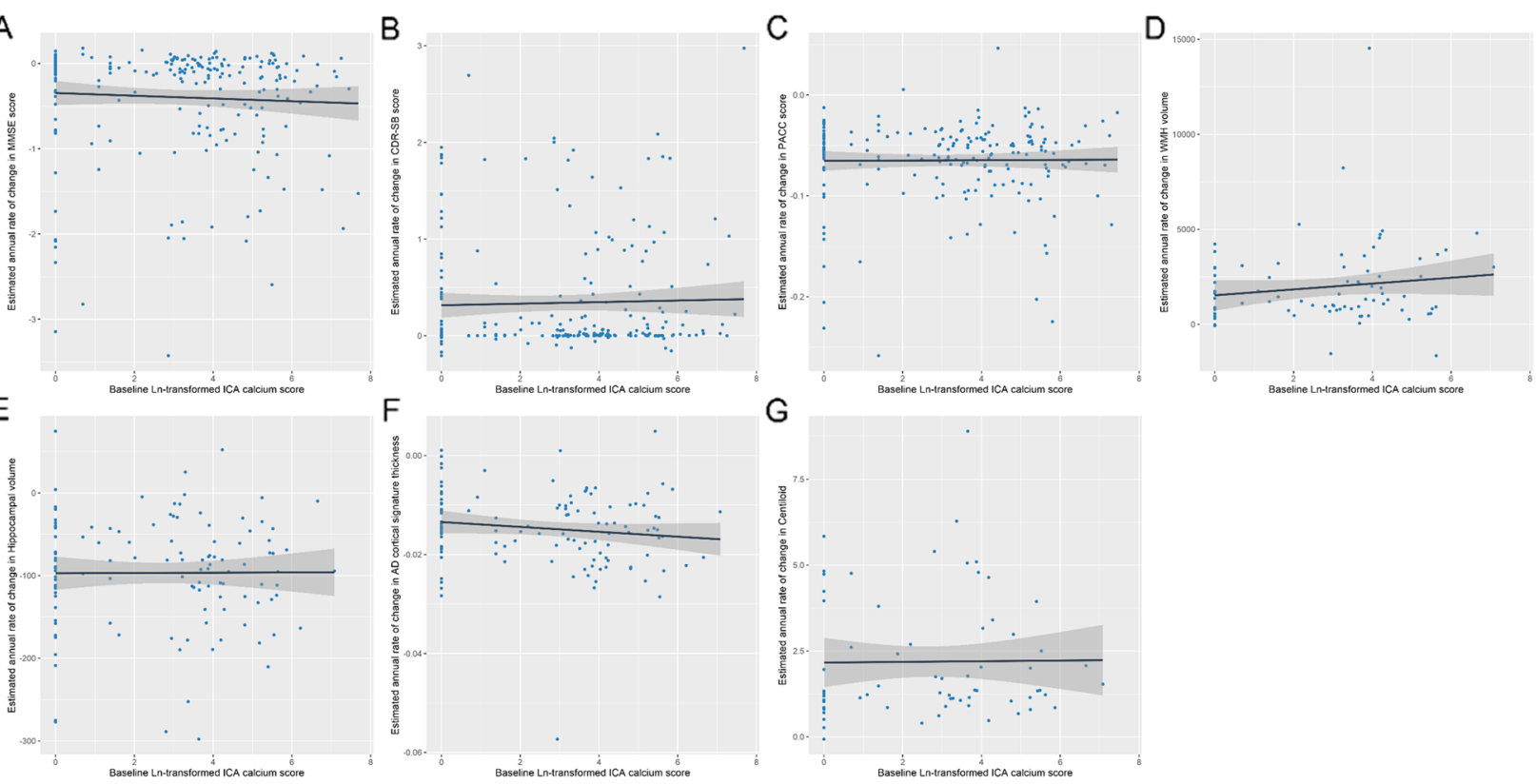

None of the models reached statistical significance. For p-values see text. ICA: internal carotid artery; MMSE: mini-mental state examination; CDR-SB: CDR Sum of Boxes score; PACC: pre-climical Alzheimer disease cognitive composite; WMH: white-matter hyperintensities; AD: Alzheimer disease; Centiloid: measure of global amyloid disposition based on conversion of PIB or AV45 PET SUVRs to a standardized scale

Fig. 3 Association of the presence of internal carotid artery calcification in baseline with longitudinal cognitive scores and AD imaging biomarkers

Table 6 Univariable linear regression model to explore the association of estimated annual rate of change of cognitive and imaging biomarkers based on baseline ICA calcium score and volume

Ln ICA calcium score as independent variable

Estimated annual rate of change in

p-value

0.957

MMSE score

CDR-SB score

PACC score

WMH volume $\left(\mathrm{mm}^{3}\right)$

Total hippocampal volume $\left(\mathrm{mm}^{3}\right)$

AD cortical signature thickness ( $\mathrm{mm}$ )

0.957

0.957

0.689

0.957

0.689

Centiloid

Ln ICA calcium volume as independent variable

Estimated annual rate of change in:

MMSE score

p-value

0.845

CDR-SB score

0.845

0.845

WMH volume $\left(\mathrm{mm}^{3}\right)$

Total hippocampal volume $\left(\mathrm{mm}^{3}\right)$

0.7

0.845

0.7

Centiloid

0.845
Coefficient (SE)

-0.016 (0.019)

$0.008(0.018)$

$1.65 \mathrm{e}-4(0.001)$

$154.2(118.5)$

$0.161(2.9)$

$-4.39 e-4(3.41 e-4)$

$0.01(0.106)$

Coefficient (SE)

$-0.015(0.02)$

$0.007(0.018)$

$4.16 e-4(1.327 \mathrm{e}-3)$

$0.153(0.118)$

$0.962(2.94)$

$-4.39 e-4(3.41 e-4)$

$0.004(0.021)$

Abbreviations: MMSE, Mini-Mental State Examination, CDR, Clinical Dementia Rating Scale, CDR-SB, Clinical Dementia Rating Scale Sum of Boxes, PACC, Preclinical Alzheimer Cognitive Composite, WMH volume, white matter hyperintensities volume, Centiloid, measure of global amyloid disposition based on conversion of PIB or AV45 PET SUVRs to a standardized scale, AD cortical signature thickness, cortical thickness in signature regions affected in Alzheimer disease (see Dincer et al. 2020), Ln ICA calcium score/volume, natural log transformation of internal carotid artery Agatston calcium score/volume, $S E$, standard error of beta coefficient

${ }^{\S} p$-values were corrected for multiple comparisons through estimation of the false discovery rate via the Benjamini and Hochberg method. Tests with a $p$-value of 0.05 were considered statistically significant in both regression models (denoted in bold) 
Table 7 Univariable linear regression model to explore the association of baseline imaging biomarkers on dementia with annual rates of change in cognitive scores

\begin{tabular}{|c|c|c|}
\hline \multirow[t]{2}{*}{ Baseline imaging biomarkers of dementia } & \multicolumn{2}{|c|}{ Estimated annual rate of change in the MMSE score } \\
\hline & $p$-value $e^{\S}$ & Coefficient (SE) \\
\hline WMH volume $\left(1000 \mathrm{~mm}^{3}\right)^{\mathrm{a}}$ & $<0.001$ & $-0.011(0.002)$ \\
\hline Total hippocampal volume $\left(100 \mathrm{~mm}^{3}\right)^{\mathrm{a}}$ & $<0.001$ & $0.036(0.004)$ \\
\hline AD cortical signature thickness (mm) & $<0.001$ & $2.36(0.257)$ \\
\hline Centiloid (5 unit) $)^{\mathrm{a}}$ & $<0.001$ & $-0.040(0.004)$ \\
\hline \multirow[t]{2}{*}{ Baseline imaging biomarkers of dementia } & \multicolumn{2}{|c|}{ Estimated annual rate of change in the PACC score } \\
\hline & $p$-value $e^{\S}$ & Coefficient (SE) \\
\hline WMH volume $\left(1000 \mathrm{~mm}^{3}\right)^{\mathrm{a}}$ & 0.169 & $-2.66 e-4(1.87 e-4)$ \\
\hline Total hippocampal volume $\left(100 \mathrm{~mm}^{3}\right)^{\mathrm{a}}$ & $<0.001$ & $0.002(0.0003)$ \\
\hline AD cortical signature thickness (mm) & $<0.001$ & $0.099(0.021)$ \\
\hline Centiloid (5 unit) $)^{\mathrm{a}}$ & $<0.001$ & $-1.969 e-3(3.45 e-4)$ \\
\hline \multicolumn{3}{|c|}{$\begin{array}{l}\text { Abbreviations: MMSE, Mini-Mental State Examination, CDR, Clinical Dementia Rating Scale, PACC, Preclinical Alzheimer Cognitive Composite, WMH volume, white } \\
\text { matter hyperintensities volume, Centiloid, measure of global amyloid disposition based on conversion of PIB or AV45 PET SUVRs to a standardized scale, AD cortical } \\
\text { signature thickness, cortical thickness in signature regions affected in Alzheimer disease (see Dincer et al. 2020), SE, standard error of beta coefficient }\end{array}$} \\
\hline \multicolumn{3}{|c|}{ a Coefficients are demonstrated for $1000-\mathrm{mm}^{3}$ increment in WMH volume, $100-\mathrm{mm}^{3}$ increment in total hippocampal volume, and 5 -unit increment in Centiloid scale } \\
\hline \multicolumn{3}{|c|}{$\begin{array}{l}{ }^{\S} p \text {-values were corrected for multiple comparisons through estimation of the false discovery rate via the Benjamini and Hochberg method. Tests with a } p \text {-value of } 0.05 \\
\text { were considered statistically significant in both regression models (denoted in bold) }\end{array}$} \\
\hline
\end{tabular}

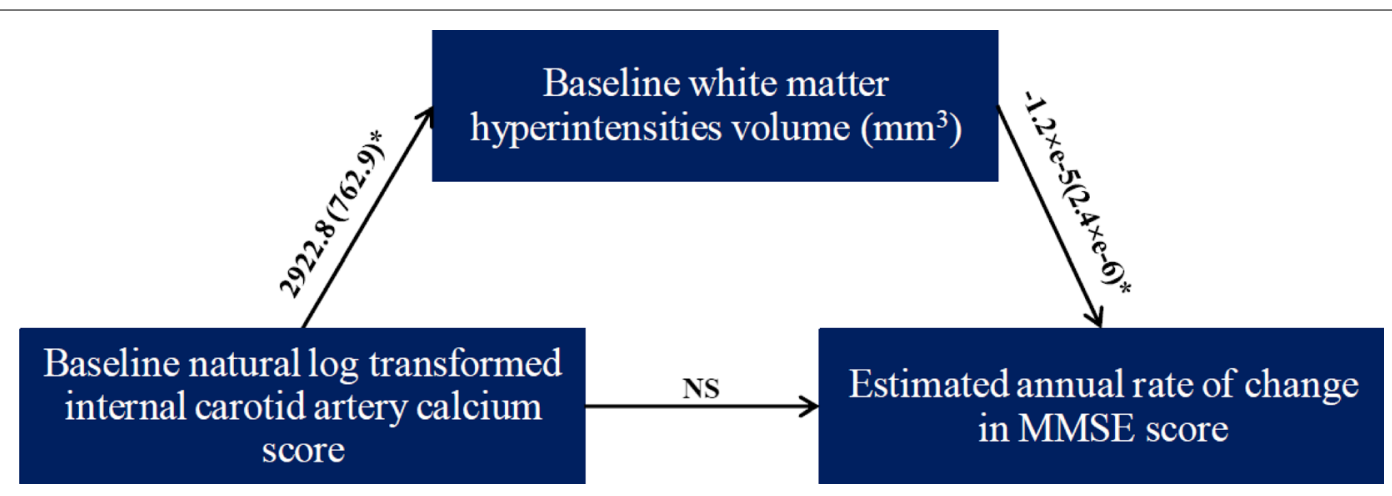

Numbers represent the regression coefficient and its standard error for each model fit. P-value for both significant models was estimated $<0.001$. MMSE: mini-mental state examination; NS: non-significant Fig. 4 Mediation model between Ln-transformed ICA calcium score and the estimated annual rate of change in the MMSE score

disease. We demonstrated (1) an independent association between increased age and male sex with the odds and severity of ICA calcification; (2) no significant difference in the odds of conversion, longitudinal cognitive scores, WMH volume, hippocampal volume, AD cortical signature thickness, or amyloid burden between participants with and without ICAC in the baseline; (3) a significant indirect effect for baseline ICAC on longitudinal MMSE score which was purely mediated via baseline WMH volume; and (4) a significant association between higher WMH volume and amyloid burden as well as lower hippocampal volume and AD cortical signature thickness in predicting lower MMSE scores in longitudinal follow-ups.
Results obtained through analysis of participants' baseline status were in line with previous literature indicating a direct relationship between age and severity of intracranial ICAC $[2,3]$. Interestingly, although ICAC was not associated with increases in WMH volume during follow-up visits, it mediated longitudinal cognition through its association with baseline WMH volume. As WMH are primarily an expression of small vessel disease and ICAC is a proxy of large vessel atherosclerosis [34], our results indicate that while both findings reflect atherosclerosis-hence correlated in severity when looked in a cross-sectional manner-they represent different clinical trajectories of atherosclerosis in different intracranial vascular beds. 
We identified no direct association between the presence or severity of ICAC and baseline or longitudinal cognitive scores (MMSE, CDR-SB, and PACC), nor did we find any association with the risk of conversion to CDR above 0 . These results were in part in agreement with findings reported by Bos et al. from the Rotterdam study where one standard deviation increase in intracranial ICAC was able to predict the risk of dementia with borderline significance ( $p$-value: 0.05$)$ and odds ratio confidence interval of close to 1 (1.34 (1.01-1.78)) [12]. Here we show in a more rigorous manner and using a longitudinally followed cohort that intracranial ICAC was unable to predict conversion to CDR > 0 even in the setting of preclinical AD. Results from the same cohort as well as a cross-sectional report from the Thai population have identified a negative association between the severity of ICAC and longitudinal changes in general (i.e., MMSE) and domain-specific cognitive scores [10-12]. None of the above studies considered or controlled for the presence of concomitant white matter lesions as a confounding factor and potential proxy of vascular dementia. Further longitudinal studies are needed to understand the effects of ICAC on specific cognitive domains rather than global cognition measured through MMSE, CDR-SB, and PACC scores in the current study.

In vivo modeling of ICAC can be achieved through direct application of calcium chloride to arterial intima in rodents [35]. Rodents with ICAC demonstrate increased pulse pressure in distal, mediumsized arteries and a resulting impaired blood flow regulation in response to neuronal activity [13]. This would lead to increased blood-brain barrier permeability, amyloid deposition, and oxidative stress in the hippocampus, followed by increased cortical and hippocampal gliosis, culminating in neurodegeneration and memory impairment in these animals [13, 35, 36]. Similar to rodents with ICA calcification, Kang et al. identified an inverse relationship between the number of stenotic intracranial arteries and hippocampal volume, but no association with total cortical amyloid burden or AD cortical signature thickness [33]. Similarly, post-mortem AD brain assessments have shown associations between amyloid pathology and reduced hippocampal volume yet no such association is demonstrated between in vivo measurement of atherosclerosis and amyloid burden in patients with $\mathrm{AD}$ $[37,38]$. When viewed in the context of animal model literature, our results present further evidence in human studies against the utility of ICAC in predicting in vivo $\mathrm{AD}$ imaging biomarkers in the setting of preclinical AD.

\section{Limitations}

While calcifications of ICA are historically regarded as being a proxy of atherosclerotic disease and hence limited to the intimal layer, a growing body of literature suggest that calcification of intracranial ICA observed on CT is predominantly non-atherosclerotic and confined in the tunica media and internal elastic lamina of the blood vessels $[3,39]$. Individuals with this medial ICAC are found to have different functional outcomes and more likely to benefit from endovascular treatment compared to those with intimal/atherosclerotic ICAC $[40,41]$. Characterization of features in ICAC that would help identify the dominant type-intimal versus medial ICAC-requires a high-resolution $\mathrm{CT}$ scan with an optimal $0.6-1-\mathrm{mm}$ slice thickness [42]. Nonetheless, the primary goal of this study was to use CT scans that have a quality similar to clinically available scans and use a tool that was available through the clinical workflow to investigate the significance of ICAC in predicting cognition. Therefore, non-contrast CT scans used for ICA calcium scoring in our study were obtained as part of the amyloid PET-CT scan performed during each participant's enrollment (slice thickness: $3 \mathrm{~mm}$ and average 40 slices per image), creating a sub-optimal resolution for detection of features corresponding to each type of ICAC. On the other hand, the proximity of intracranial ICA to the petrous bone and bony skull base including the clinoid process limited our ability to delineate regions of interest around calcifications without including bone in the cavernous and clinoid segments of ICA. Differentiation between different ICAC subtypes and calcium scoring based on ICA segments were therefore beyond the sensitivity of the $\mathrm{CT}$ scans used in our study. Future studies should address this limitation through a prospective gathering of high-resolution CT scan data in the baseline and as part of follow-up imaging.

Importantly, the sample size pool for our longitudinal data points was different among different biomarker categories (Table 5), with the lowest number of data points and participants in the longitudinal Centiloid measures. We addressed this issue using linear mixed models to estimate the annual rates of longitudinal outcomes, a method known to be relatively unbiased in the presence of missing data [43].

Although ICA calcium score or volume did not show any predictive value for general cognitive outcomes in our longitudinal follow-up, cross-sectional data from the Rotterdam Study show evidence of an association between intracranial ICAC and worse performance in executive function, information processing speed, and motor speed domains [44]. These are among domains commonly associated with WM disease in the elderly population [45]. It is therefore imperative for future longitudinal studies to 
investigate the association of ICAC with changes in ADspecific cognitive domains. Last but not least, despite ongoing efforts in the Knight ADRC to enroll and more diverse and representative cohort of older adults, our study participants were primarily Caucasian.

\section{Conclusions}

ICAC is frequently identified as an incidental finding on head CT scan and physicians are often uncertain about its prognostic and diagnostic value of such finding. Together, our results reveal that intracranial ICAC is unable to predict the onset of dementia in cognitively normal adults, nor it is able to predict longitudinal changes in cognitive scores, imaging makers of small vessel disease such as WMH volume, or AD imaging biomarkers. ICAC might however have an indirect effect on longitudinal cognition that is mediated by its effect on white matter disease. WMH volume might therefore be predictive of cognitive outcomes and hence an important incidental finding to report.

\section{Acknowledgements}

Not applicable

\section{Authors' contributions}

The study was conceived by FR, MN, and TLSB. HLPO, TLSB, and JCM were responsible for funding and data acquisition. Analysis design was conceived, conducted, and interpreted by FR, CDC, NM, and AD with direct supervision from GC and JL. FR and MN contributed to the composition of the manuscript, and all authors contributed to the manuscript preparation and provided substantial revision. All authors have approved the submitted version and have agreed both to be personally accountable for the author's own contributions and to ensure that questions related to the accuracy or integrity of any part of the work are appropriately investigated, resolved, and the resolution documented in the literature.

\section{Funding}

This research was supported by the following: ADRC Center Grant, NIH/ NIA P50AG005681, The Barnes-Jewish Hospital Foundation (BJHF), BarnesJewish Hospital Foundation (PIB imaging), NIH/NIA P01AG026276, NIA grant T32AG05851804 (M. Nguyen), generous support from Fred Simmons and Olga Mohan, The McDonnell Center for Systems Neuroscience, 223922 26239N, NIH/NIA P01AG003991, Avid Radiopharmaceuticals, a wholly owned subsidiary of Eli Lilly (florbetapir and AV1451 imaging). For florbetapir (AV45), Avid pharmaceuticals provided the doses and partial support for scanning through an investigator-initiated research grant awarded to Washington University (J.C. Morris, T.L.S. Benzinger). Additional support for image acquisition was provided by CCIR/ICTS Human Imaging Unit (NIH/NCATS UL1TR000448), and support for imaging informatics (CNDA/XNAT) was provided in part by the Neuroimaging Informatics and Analysis Center (1P30NS098577) and R01 EB009352.

\section{Availability of data and materials}

De-identified participant data including clinical and cognitive assessment, CSF biomarkers, and dMRI images are available upon request through the Knight ADRC Leadership Committee (https://knightadrc.wustl.edu/research/resou rcerequest.htm).

\section{Declarations}

\section{Ethics approval and consent to participate}

The institutional ethics review board of Washington University School of Medicine in Saint Louis approved the protocol of this study. All participants consented to participate in the study and provided their informed consent to participate in the study. The study was in compliance with HIPAA and was performed in accordance with the Declaration of Helsinki.

\section{Consent for publication}

Not applicable

\section{Competing interests}

Dr. Benzinger has investigator-initiated research funding from the $\mathrm{NIH}$, the Alzheimer's Association, the Barnes-Jewish Hospital Foundation, and Avid Radiopharmaceuticals (a wholly owned subsidiary of Eli Lilly). Dr. Benzinger participates as a site investigator in clinical trials sponsored by Avid Radiopharmaceuticals, Eli Lilly, Biogen, Eisai, Jansen, and Roche; is an unpaid consultant to Eisai and Siemens; and is on an Advisory Board and Speaker's Bureau for Biogen.

\section{Author details}

'Mallinckrodt Institute of Radiology, Washington University School of Medicine, St. Louis, 510 South Kingshighway Boulevard, Campus Box 8131, St. Louis, MO 63110, USA. ${ }^{2}$ Charles F. and Joanne Knight Alzheimer Disease Research Center (Knight ADRC), Washington University, St. Louis, MO, USA. ${ }^{3}$ Department of Neurology, Washington University in Saint Louis, St. Louis, MO, USA. ${ }^{4}$ Division of Public Health Sciences, Department of Surgery, Washington University School of Medicine (WUSM), St. Louis, MO, USA.

Received: 15 September 2021 Accepted: 31 January 2022

Published online: 11 February 2022

References

1. Berliner JA, Navab M, Fogelman AM, Frank JS, Demer LL, Edwards PA, et al. Atherosclerosis: basic mechanisms. Oxidation, inflammation, and genetics. Circulation. 1995;91(9):2488-96.

2. Bos D, Portegies MLP, van der Lugt A, Bos MJ, Koudstaal PJ, Hofman A, et al. Intracranial carotid artery atherosclerosis and the risk of stroke in whites: the Rotterdam Study. JAMA Neurol. 2014;71(4):405-11.

3. Kockelkoren R, De Vis JB, de Jong PA, Vernooij MW, Mali WPTM, Hendrikse J, et al. Intracranial carotid artery calcification from infancy to old age. J Am Coll Cardiol. 2018;72(5):582-4 Available from: https://www.sciencedir ect.com/science/article/pii/S0735109718349039.

4. Bartstra JW, van den Beukel TC, Van Hecke W, Mali WPTM, Spiering W, Koek HL, et al. Intracranial arterial calcification: prevalence, risk factors, and consequences: JACC review topic of the week. J Am Coll Cardiol. 2020;76(13):1595-604 Available from: https://www.sciencedirect.com/ science/article/pii/S0735109720361726.

5. Erbay S, Han R, Baccei S, Krakov W, Zou KH, Bhadelia R, et al. Intracranial carotid artery calcification on head CT and its association with ischemic changes on brain $\mathrm{MRI}$ in patients presenting with stroke-like symptoms: retrospective analysis. Neuroradiology. 2007;49(1):27-33.

6. Chen Y-C, Wei X-E, Lu J, Qiao R-H, Shen X-F, Li Y-H. Correlation between intracranial arterial calcification and imaging of cerebral small vessel disease. Front Neurol. 2019;10:426 Available from: https://pubmed.ncbi. nlm.nih.gov/31118918.

7. Bos D, Ikram MA, Elias-Smale SE, Krestin GP, Hofman A, Witteman JCM, et al. Calcification in major vessel beds relates to vascular brain disease. Arterioscler Thromb Vasc Biol. 2011:31(10):2331-7.

8. Hong NR, Seo HS, Lee YH, Kim JH, Seol HY, Lee NJ, et al. The correlation between carotid siphon calcification and lacunar infarction. Neuroradiology. 2011;53(9):643-9.

9. Bolandzadeh N, Davis JC, Tam R, Handy TC, Liu-Ambrose T. The association between cognitive function and white matter lesion location in older adults: a systematic review. BMC Neurol. 2012;12(1):126. https://doi. org/10.1186/1471-2377-12-126.

10. Bos D, Vernooij MW, Elias-Smale SE, Verhaaren BFJ, Vrooman HA, Hofman $A$, et al. Atherosclerotic calcification relates to cognitive function and to brain changes on magnetic resonance imaging. Alzheimers Dement. 2012;8(5, Supplement):S104-11 Available from: https://www.sciencedir ect.com/science/article/pii/S1552526012000283.

11. Kao H-W, Liou M, Chung H-W, Liu H-S, Tsai P-H, Chiang S-W, et al. High Agatston calcium score of intracranial carotid artery: a significant risk 
factor for cognitive impairment. Medicine (Baltimore). 2015;94(39):e1546 Available from: https://pubmed.ncbi.nlm.nih.gov/26426620.

12. Bos D, Vernooij MW, de Bruijn RFAG, Koudstaal PJ, Hofman A, Franco OH, et al. Atherosclerotic calcification is related to a higher risk of dementia and cognitive decline. Alzheimers Dement. 2015;11(6):639-47 e1.

13. Muhire G, Iulita MF, Vallerand D, Youwakim J, Gratuze M, Petry FR, et al. Arterial stiffness due to carotid calcification disrupts cerebral blood flow regulation and leads to cognitive deficits. J Am Heart Assoc. 2019;8(9):e011630 Available from: https://pubmed.ncbi.nlm.nih.gov/ 31057061.

14. Morris JC. The Clinical Dementia Rating (CDR). Neurology. 1993;43(11):2412 LP-2412-a Available from: http://n.neurology.org/conte $\mathrm{nt} / 43 / 11 / 2412.2$.abstract.

15. McKhann GM, Knopman DS, Chertkow H, Hyman BT, Jack Jr CR, Kawas $\mathrm{CH}$, et al. The diagnosis of dementia due to Alzheimer's disease: recommendations from the National Institute on Aging-Alzheimer's Association workgroups on diagnostic guidelines for Alzheimer's disease. Alzheimers Dement. 2011;7(3):263-9. Available from: https://pubmed.ncbi.nlm.nih. gov/21514250.

16. O'Bryant SE, Waring SC, Cullum CM, Hall J, Lacritz L, Massman PJ, et al. Staging dementia using Clinical Dementia Rating Scale Sum of Boxes scores: a Texas Alzheimer's research consortium study. Arch Neurol. 2008;65(8):1091-5 Available from: https://pubmed.ncbi.nlm.nih.gov/ 18695059.

17. Donohue MC, Sperling RA, Salmon DP, Rentz DM, Raman R, Thomas RG, et al. The preclinical Alzheimer cognitive composite: measuring amyloidrelated decline. JAMA Neurol. 2014;71(8):961-70 Available from: https:// pubmed.ncbi.nlm.nih.gov/24886908.

18. Agatston AS, Janowitz WR, Hildner FJ, Zusmer NR, Viamonte MJ, Detrano R. Quantification of coronary artery calcium using ultrafast computed tomography. J Am Coll Cardiol. 1990;15(4):827-32.

19. Neves PO, Andrade J, Monção H. Coronary artery calcium score: current status. Radiol Bras. 2017;50(3):182-9 Available from: https://pubmed.ncbi. nlm.nih.gov/28670030.

20. SuY, D'Angelo GM, Vlassenko AG, Zhou G, Snyder AZ, Marcus DS, et al. Quantitative analysis of PiB-PET with FreeSurfer ROls. PLoS One. 2013;8(11):e73377.

21. Su Y, Blazey TM, Snyder AZ, Raichle ME, Marcus DS, Ances BM, et al. Partial volume correction in quantitative amyloid imaging. Neuroimage. 2015;107:55-64.

22. Su Y, Flores S, Hornbeck RC, Speidel B, Vlassenko AG, Gordon BA, et al. Utilizing the Centiloid scale in cross-sectional and longitudinal PiB PET studies. Neurolmage Clin. 2018;19:406-16 Available from: https://pubmed.ncbi.nlm.nih.gov/30035025.

23. Schmidt P, Gaser C, Arsic M, Buck D, Förschler A, Berthele A, et al. An automated tool for detection of FLAIR-hyperintense white-matter lesions in multiple sclerosis. Neuroimage. 2012;59(4):3774-83 Available from: https://www.sciencedirect.com/science/article/pii/S1053811911013139.

24. Fischl B, Salat DH, Busa E, Albert M, Dieterich M, Haselgrove C, et al. Whole brain segmentation: automated labeling of neuroanatomical structures in the human brain. Neuron. 2002;33(3):341-55.

25. Dincer A, Gordon BA, Hari-Raj A, Keefe SJ, Flores S, McKay NS, et al. Comparing cortical signatures of atrophy between late-onset and autosomal dominant Alzheimer disease. Neurolmage Clin. 2020;28:102491 Available from: https://www.sciencedirect.com/science/article/pii/S221315822 0303284

26. Benjamini Y, Hochberg Y. Controlling the false discovery rate: a practical and powerful approach to multiple testing. J R Stat Soc Ser B. 1995;57(1):289-300. https://doi.org/10.1111/j.2517-6161.1995.tb02031.x.

27. Chowdhury MZI, Turin TC. Variable selection strategies and its importance in clinical prediction modelling. Fam Med Community Heal. 2020;8(1):e000262 Available from: https://pubmed.ncbi.nlm.nih.gov/ 32148735.

28. Tingley D, Yamamoto T, Hirose K, Keele L, Imai K. mediation: R package for causal mediation analysis. J Stat Softw. 2014;59(5):1-38.

29. den Heijer T, van der Lijn F, Koudstaal PJ, Hofman A, van der Lugt A, Krestin GP, et al. A 10-year follow-up of hippocampal volume on magnetic resonance imaging in early dementia and cognitive decline. Brain. 2010;133(Pt 4):1163-72.

30. Dickerson BC, Wolk DA, Initiative ADN. MRI cortical thickness biomarker predicts AD-like CSF and cognitive decline in normal adults. Neurology.
2012;78(2):84-90 Available from: https://pubmed.ncbi.nlm.nih.gov/ 22189451.

31. Farrell ME, Kennedy KM, Rodrigue KM, Wig G, Bischof GN, Rieck JR, et al. Association of longitudinal cognitive decline with amyloid burden in middle-aged and older adults: evidence for a dose-response relationship. JAMA Neurol. 2017;74(7):830-8. https://doi.org/10.1001/jamaneurol.2017. 0892.

32. Wu XH, Chen XY, Wang $\sqcup$, Wong KS. Intracranial artery calcification and its clinical significance. J Clin Neurol. 2016;12(3):253-61 Available from: https://pubmed.ncbi.nlm.nih.gov/27165425.

33. Kang KM, Byun MS, Lee JH, Yi D, Choi HJ, Lee E, et al. Association of carotid and intracranial stenosis with Alzheimer's disease biomarkers. Alzheimers Res Ther. 2020;12(1):106. https://doi.org/10.1186/s13195-020-00675-6.

34. Wardlaw JM, Valdés Hernández MC, Muñoz-Maniega S. What are white matter hyperintensities made of? Relevance to vascular cognitive impairment. J Am Heart Assoc. 2015;4(6):1140 Available from: https://pubmed. ncbi.nlm.nih.gov/26104658.

35. Nataliya S, Diane V, Edgar G, Frédéric L, Hélène G. Carotid calcification in mice: a new model to study the effects of arterial stiffness on the brain. J Am Heart Assoc. 2021;2(3):e000224. https://doi.org/10.1161/JAHA.113. 000224.

36. Winder NR, Reeve EH, Walker AE. Large artery stiffness and brain health: insights from animal models. Am J Physiol Circ Physiol. 2020;320(1):H42431. https://doi.org/10.1152/ajpheart.00696.2020.

37. Gottesman RF, Mosley TH, Knopman DS, Hao Q, Wong D, Wagenknecht $L E$, et al. Association of intracranial atherosclerotic disease with brain $\beta$-amyloid deposition: secondary analysis of the ARIC study. JAMA Neurol. 2020;77(3):350-7. https://doi.org/10.1001/jamaneurol.2019.4339.

38. Yarchoan M, Xie SX, Kling MA, Toledo JB, Wolk DA, Lee EB, et al. Cerebrovascular atherosclerosis correlates with Alzheimer pathology in neurodegenerative dementias. Brain. 2012;135(12):3749-56. https://doi.org/10. 1093/brain/aws271.

39. Vos A, Van Hecke W, Spliet WGM, Goldschmeding R, Isgum I, Kockelkoren $\mathrm{R}$, et al. Predominance of nonatherosclerotic internal elastic lamina calcification in the intracranial internal carotid artery. Stroke. 2016 Jan; $47(1): 221-3$.

40. Luijten SPR, van der Donk SC, Compagne KCJ, Yo LSF, Sprengers MES, Majoie CBLM, et al. Intracranial carotid artery calcification subtype and collaterals in patients undergoing endovascular thrombectomy. Atherosclerosis. 2021;337:1-6 Available from: https://www.sciencedirect.com/ science/article/pii/S0021915021013897.

41. Compagne KCJ, Clephas PRD, Majoie CBLM, Roos YBWEM, Berkhemer $\mathrm{OA}$, van Oostenbrugge RJ, et al. Intracranial carotid artery calcification and effect of endovascular stroke treatment. Stroke. 2018;49(12):2961-8 Available from: https://pubmed.ncbi.nlm.nih.gov/30571406.

42. Kockelkoren R, Vos A, Van Hecke W, Vink A, Bleys RLAW, Verdoorn D, et al. Computed tomographic distinction of intimal and medial calcification in the intracranial internal carotid artery. PLoS One. 2017;12(1):e0168360.

43. Ibrahim JG, Molenberghs G. Missing data methods in longitudinal studies: a review. Test (Madr). 2009;18(1):1-43 Available from: https://pubmed. ncbi.nlm.nih.gov/21218187.

44. Bos D, van der Rijk MJM, Geeraedts TEA, Hofman A, Krestin GP, Witteman $J C M$, et al. Intracranial carotid artery atherosclerosis: prevalence and risk factors in the general population. Stroke. 2012 Jul;43(7):1878-84.

45. Vernooij MW, Ikram MA, Vrooman HA, Wielopolski PA, Krestin GP, Hofman $A$, et al. White matter microstructural integrity and cognitive function in a general elderly population. Arch Gen Psychiatry. 2009;66(5):545-53. https://doi.org/10.1001/archgenpsychiatry.2009.5.

\section{Publisher's Note}

Springer Nature remains neutral with regard to jurisdictional claims in published maps and institutional affiliations. 